\title{
Stochastic Growth with Increasing Returns: Stability and Path Dependence
}

\author{
John Stachurski \\ Institute of Economic Research, Kyoto University, Yoshida-honmachi \\ Sakyo-ku, Kyoto 606-8501, Japan
}

\begin{abstract}
The paper demonstrates global stability in a class of stochastic overlapping generations economies with increasing returns. These results are applied to the study of path dependent dynamics. In particular, for nonlinear stochastic models it is seen that persistence of the historical state and formal ergodicity may easily coincide. A new definition of path dependence is proposed that incorporates such dynamics.
\end{abstract}

Key words: Increasing returns, path dependence, ergodicity.

\section{Introduction}

It is well-known that external production economies can generate multiple long-run equilibria in models of economic development. ${ }^{2}$ In such models, outcomes are not uniquely determined by the fundamental structure, but depend also on the historical state of the process. Here, low level equilibria have the interpretation of a poverty trap.

In this paper it is shown that shocks may induce uniqueness of stochastic steady state and global asymptotic stability for developing economies typically

Email address: john@kier.kyoto-u.ac.jp (John Stachurski).

1 The author thanks John Creedy, Kazuo Nishimura, Rabee Tourky and Cuong Le Van for helpful comments; Centre de Recherche en Mathématiques, Statistique et Economie Mathématique, Université Paris 1, Panthéon-Sorbonne for their hospitality during a research visit, and the Japan Society for the Promotion of Science for financial support.

2 See, for example, Azariadis and Drazen (1990), Galor and Ziera (1993), Quah (1996), David (1997) and Acemoglu and Zilibotti (1997). 
associated with persistence and sensitivity to initial conditions. (The notion of stochastic steady state is the standard one of Brock and Mirman, 1972.) The model is that of Azariadis and Drazen (1990), with a stochastic component included in production.

Our results are applied to the study of path dependence. Since the work of Arthur (1994) and David (1997) in particular, interest in the notion of path dependent evolution has been strong. These and subsequent authors consider models with positive feedback due to increasing returns. Contrary to the homeostasis encountered when returns diminish, positive feedback generates local persistence, phase transitions and switching behavior, all of which appear useful to replicate macroeconomic time series.

The basic idea behind path dependence is that long-run outcomes depend on initial conditions; a plurality of local attractors serve to lock in the attributes of the historical state. In this connection, Arthur (1994, p. 13) defines a model to be path dependent whenever it is not ergodic. Alternatively, David (1997, p. 14) defines a path dependent process to be one "whose asymptotic distribution evolves as a consequence of the processes' own history." He cites "multiplicity of absorbing states" as a source of this outcome.

In this paper we exhibit standard increasing returns models that are both arbitrarily persistent (in a sense made precise) and formally ergodic. There is only one (minimal) absorbing state. Thus none of the above definitions for path dependence are satisfied. These findings indicate that the standard definitions may be too narrow.

The central problem is that long-run behavior of a nonlinear random system can be counterintuitive when one passes to the infinite limit. In this connection, we propose an alternative definition based on finite statistical properties. In short, an economic process is called path dependent if there are multiple disjoint "metastable" sets. A second advantage of this approach is that the statement concerns model primitives rather than the ergodic distribution, the properties of which are often difficult to verify.

\section{A Stochastic Model with Externalities}

Consider the following overlapping generations economy. There is a single, perishable good which can be either consumed or used as capital input for production. Agents live for two periods, working in the first and living off savings in the second. Savings in the first period forms capital stock, which in the following period is combined with the labor of a new generation of young agents for production under the technology $y_{t}=A_{t} F\left(k_{t}, \ell_{t}\right) \varepsilon_{t}^{\sigma}$. 
Here $y$ is aggregate output, $k$ is the stock of physical capital, $\ell$ is labor input, $F$ is a homogenous-of-degree-one function and $\varepsilon$ is a stochastic component. For convenience, we set $\ell_{t}=1$ for all $t$, and define $f(k):=F(k, 1)$.

The exponent $\sigma \geq 0$ parameterizes the amplitude of the shock. We admit the possibility that $\sigma=0$, in which case the model is deterministic. Note also that the scale parameter $A_{t}$ is nonstationary.

In period zero there is a generation of already old, with total savings $k_{0}$. At the start of each period the shock $\varepsilon$ is selected by nature and revealed. Production then takes place.

All of the following conditions on production technology are standard.

Assumption 2.1 The function $f$ satisfies $f(0)=0$; is twice differentiable on $(0, \infty), f^{\prime}>0, f^{\prime \prime}<0$; and $\lim _{x \downarrow 0} f^{\prime}(x)=\infty, \lim _{x \uparrow \infty} f^{\prime}(x)=0$.

In the overlapping generations model, additional assumptions are required to prevent the economy collapsing to zero output. Assumption 2.1 implies that competitive factor payments to capital $k f^{\prime}(k)$ are strictly less than total income $f(k)$. Assume this bound is uniform:

Assumption 2.2 There exists a $\lambda<1$ such that $k f^{\prime}(k) \leq \lambda f(k), \forall k>0$.

Regarding the shock, which is common to all producers,

Assumption 2.3 The sequence $\left(\varepsilon_{t}\right)$ is uncorrelated, and $\ln \varepsilon_{t} \sim \mathrm{N}(0,1)$.

Let $c$ (resp. $c^{\prime}$ ) denote consumption while young (resp. old). All consumer preferences have the specification

$$
U\left(c_{t}, c_{t+1}^{\prime}\right)=\ln c_{t}+\beta \mathbb{E} \ln c_{t+1}^{\prime},
$$

which agents maximize subject to

$$
c_{t+1}^{\prime} \leq\left(w_{t}-c_{t}\right)\left(1+r_{t+1}\right) .
$$

Here $\beta \in(0,1)$ is a discount factor, and $w_{t}$ and $r_{t}$ are the wage and interest rates at $t$ respectively.

Assume that depreciation is total, in which case savings, investment and next period capital stock are all equal. A competitive equilibrium for the economy is a nonnegative sequence $\left(k_{t}, c_{t}, c_{t}^{\prime}, r_{t}, w_{t}\right)_{t \geq 0}$ such that $k_{0}$ is initial savings, and for $t=0,1, \ldots$,

1. $c_{t}+c_{t}^{\prime}+k_{t+1}=A_{t} f\left(k_{t}\right) \varepsilon_{t}^{\sigma}$,

2. $1+r_{t}=A_{t} f^{\prime}\left(k_{t}\right) \varepsilon_{t}^{\sigma}$, 




Fig. 1. Threshold Externalities.

3. $w_{t}=A_{t} f\left(k_{t}\right) \varepsilon_{t}^{\sigma}-k_{t} A_{t} f^{\prime}\left(k_{t}\right) \varepsilon_{t}^{\sigma}$, and

4. $\left(c_{t}, c_{t+1}^{\prime}\right)$ maximizes (1) subject to $(2)$.

The first condition is one of material balance; $2-3$ dictate competitive factor payments under profit maximization. The last - utility maximization - implies a savings rate from wage income of $\beta /(1+\beta)$.

It remains to specify the process $\left(A_{t}\right)$. In the tradition of endogenous growth, it is supposed that technology depends on economy-wide aggregates; in this case the capital stock $k$. Thus $A_{t}=A\left(k_{t}\right)$. The influence of these spillovers on the economy may be highly nonlinear. In this paper we require only that

Assumption 2.4 The function $k \mapsto A(k)$ is Borel-measurable and takes values in a compact subset of $(0, \infty)$.

The equilibrium law of motion for capital is easily shown to be

$$
k_{t+1}=S\left(k_{t}\right) \varepsilon_{t}^{\sigma}
$$

where $S(k):=D A(k)\left[f(k)-k f^{\prime}(k)\right]$, and $D:=\beta /(1+\beta)$.

Example 1 Let $f(k)=k^{\alpha}, \alpha \in(0,1)$. Following Azariadis and Drazen (1990), assume that $k \mapsto A(k)$ is a step function with discontinuity at a critical "threshold" value of capital per head $k_{b}$. Specifically,

$$
A(k)=A_{1} \cdot \mathbf{1}_{\left[0, k_{b}\right)}(k)+A_{2} \cdot \mathbf{1}_{\left[k_{b}, \infty\right)}(k),
$$

where $\mathbf{1}_{B}$ denotes the indicator function of set $B$. The idea is that at some value $k_{b}$, physical or human capital infrastructure achieves a critical mass, allowing rapid increase in social returns to scale.

Let $k_{i}^{*}$ be the unique solution to $k=D A_{i}(1-\alpha) k^{\alpha}$ for $i=1,2$. Assume further that $k_{1}^{*}<k_{b}<k_{2}^{*}$. A plot of the deterministic part $S(k)$ of (3) is given 
in Figure 1. The two fixed points $k_{1}^{*}$ and $k_{2}^{*}$ act as local attractors for disjoint open neighborhoods.

\section{Long-Run Dynamics}

The sequence $\left(k_{t}\right)$ is a Markov process on the state space $(0, \infty) .{ }^{3}$ Let $\varphi_{t}$ denote the marginal distribution of $k_{t} \cdot{ }^{4}$ A sequence $\left(\varphi_{t}\right)$ such that $k_{t}$ obeys (3) is called a trajectory for the economy. Each initial condition $k_{0} \in(0, \infty)$ determines a well-defined trajectory.

A long-run equilibrium or stochastic steady state is a distribution $\varphi^{*}$ such that if $k_{t} \sim \varphi^{*}$ then $k_{t+j} \sim \varphi^{*}$ for all $j \geq 0$. A stochastic steady state is called globally stable or ergodic if the trajectory $\left(\varphi_{t}\right)$ converges to $\varphi^{*}$ in a suitable topology, for every initial condition $k_{0}>0$. In this paper the topology of convergence is the strong topology induced by the total variation norm.

Since the model assumptions incorporate highly nonlinear dynamics and multiple local attractors in the deterministic case, it is somewhat surprising that

Theorem 1 For every $\sigma>0$, the stochastic process (3) has a unique stochastic steady state. The steady state is globally stable in the norm topology.

Uniqueness is completely trivial, given that the support of the shock $\varepsilon$ is equal to the entire state space. Such Markov chains always have at most one steady state distribution. Existence and global stability are not trivial. For example, none of the 3 conditions - compactness, continuity and monotonicity - in the familiar technique of Stokey, Lucas and Prescott (1989, Section 12.4) is satisfied. However, it turns out that the absolute continuity of the shock has strong regularizing properties that compensate for the weakness of Assumption 2.4, which admits highly irregular behavior. In addition, Assumption 2.2 on $f$, combined with the small left tail of the shock, mean that the economy will not collapse to zero, while the boundedness of $k \mapsto A(k)$ and the usual decreasing returns on $f$-combined with a small right tail on the shock - are enough to ensure that the economy does not grow without bounds. The details are in Section 5.

Incidentally, Theorem 1 is certainly not the first result to show that noise is potentially stabilizing. For example, in physical systems Mackey et al. (1990) demonstrate global stability when additive or multiplicative Gaussian white noise is added to the Fokker-Planck differential equation.

$\overline{3}$ Here zero is excluded from the state space to eliminate the trivial steady state where all variables are zero in each period.

4 The distribution $\varphi_{t}$ is a probabilistic measure on the Borel sets of $(0, \infty)$. 


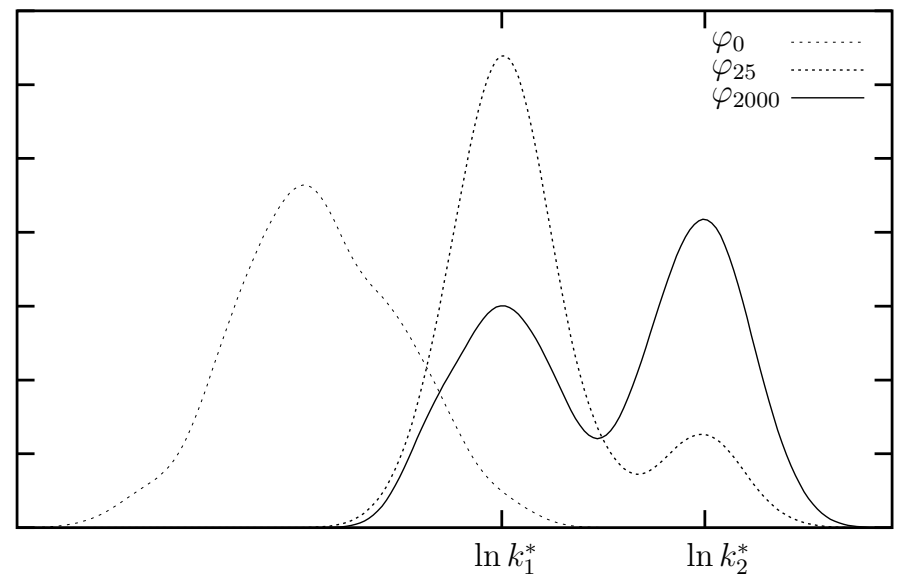

Fig. 2. Convergence to Equilibrium

Theorem 1 is illustrated in Figure 2, which shows a sequence of probability distributions (densities) generated by the economy described in Example 1. The figure can be interpreted as follows. The horizontal axis is (the log of) capital per head. The density $\varphi_{0}$, which is the left-most density in the figure, is an arbitrary starting point. The sequence of distributions moving forward in time is generated by Monte Carlo simulation on (3), and fitted with nonparametric smoothing kernels. ${ }^{5}$ For this particular initial distribution, probability mass shifts rightwards, indicating that income grows on average.

The maxima of the two modes of the approximate limit $\varphi_{2000}$ correspond to the (log of the) two local attractors $k_{1}^{*}$ and $k_{2}^{*}$ in the deterministic case (see Figure 1). Thus economies still tend on average to one of the deterministic attractors. Occasionally they make the transition between modes. In physics these regime shifts are referred to as "phase transitions," while in growth theory they are called "take-offs," "growth miracles" and "growth disasters."

\section{Path Dependence}

Given that Theorem 1 holds for any $\sigma>0$, even arbitrarily small amounts of noise induce global stability; in the infinite limit, only one long run outcome is consistent with a given set of economic fundamentals. In particular, no economy in this class is path dependent according to the definitions of Arthur and David (see Section 1). On the other hand, the deterministic version (i.e., $\sigma=0$ ) may have multiple, history-dependent steady states, as in Figure 1. It appears likely, then, that when $\sigma$ is almost zero, high degrees of persistence may be observed.

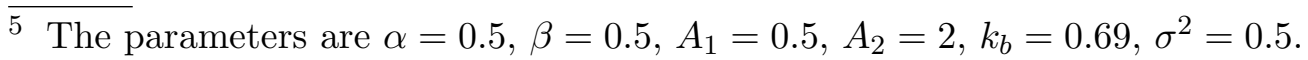




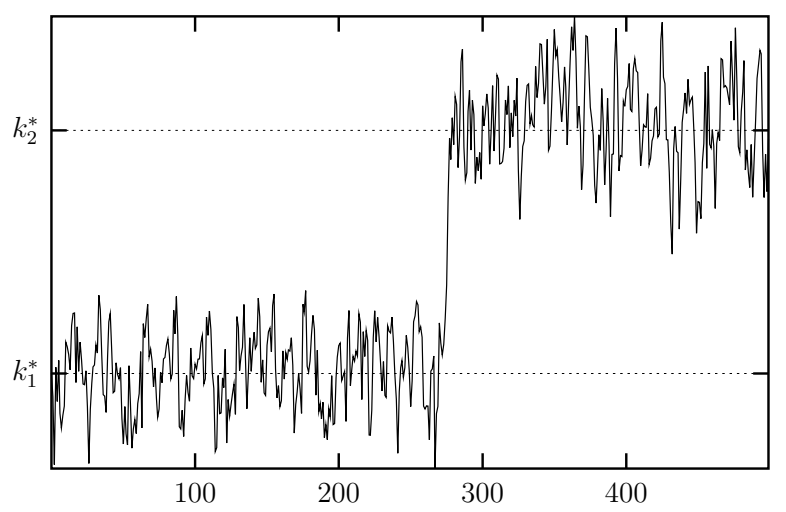

Fig. 3. Persistence in Time Series

Indeed this is the case. Consider the Azariadis-Drazen economy of Example 1. A simulated run is given in Figure 3. The horizontal axis is time, and the vertical axis is the state variable $k$. This simulation run spends nearly 300 generations in the poverty trap before making the transition to the higher equilibrium. The transition takes place as the result of a particularly fortuitous shock. ${ }^{6}$

The point is not whether the parameters that generate such a run are realistic. The point is a logical one: Given any $\eta>0$ and any finite time horizon $T$, it is possible to reduce the amplitude of the noise so that the probability of leaving the poverty trap prior to time $T$ is less than $\eta$. In our view, any model with this property should be classified as path dependent, regardless of ergodicity in the infinite limit. Indeed, this property might be used to characterize path dependence.

To formalize the problem, define a function $(k, B) \mapsto \mathbf{M}_{\sigma}(k, B)$ from $(0, \infty) \times$ $\mathscr{B}$, where $\mathscr{B}$ is the Borel sets, to $[0,1]$ by

$$
\mathbf{M}_{\sigma}(k, B):=\int \mathbf{1}_{B}\left[S(k) z^{\sigma}\right] \psi(z) d z
$$

where, as before, $\mathbf{1}_{B}$ is the indicator function, $S$ is the function in (3), and $\psi$ is the density of the shock $\varepsilon$ (i.e., the lognormal distribution). In this case, $\mathbf{M}_{\sigma}(k, B)$ is interpreted as the probability that the capital stock takes values in $B$ next period, given that it is currently equal to $k$.

A set $\Lambda \in \mathscr{B}$ is called absorbing for the process (3) whenever

$$
\mathbf{M}_{\sigma}\left(k, \Lambda^{c}\right)=0, \quad \forall k \in \Lambda,
$$

where $\Lambda^{c}:=(0, \infty) \backslash \Lambda$. Absorbing sets have the property that, once entered,

$\overline{6}$ The parameters are $\alpha=0.5, \beta=0.9, A_{1}=20, A_{2}=24, \sigma=0.05, k_{b}=3.23$. The output is displayed in logs. 
the probability of exit next period — or, indeed, at any future time - is zero. ${ }^{7}$

David (1997) mentions both multiplicity of absorbing states and lack of ergodicity in defining path dependence (see above). Before going on let us clarify the relationship between these concepts.

Proposition 2 If multiple disjoint absorbing sets exist, then (3) is not ergodic.

A proof is given in Section 5 .

The objective of this section is to develop a more inclusive definition of path dependence that captures highly persistent models such as the ones investigated in this paper. Following Huisinga (2001), and by analogy with the definition of absorbing sets,

Definition 3 Set $\Lambda \in \mathscr{B}$ is called metastable for the economy (3) if

$$
\mathbf{M}_{\sigma}\left(k, \Lambda^{c}\right) \rightarrow 0 \text { uniformly in } k \text { as } \sigma \rightarrow 0 \text {. }
$$

A subset of the state space is metastable if the maximum probability of exit in one step becomes arbitrarily small at low levels of noise. Using the concept of metastability, the following definition of path dependence suggests itself.

Definition 4 The economy (3) is defined to be path dependent whenever there exist multiple nonempty closed intervals that are disjoint and metastable.

This definition of path dependence includes the stochastic Azariadis-Drazen model:

Proposition 5 The Azariadis-Drazen economy in Example 1 is path dependent in the sense of Definition 4.

Proof Two disjoint metastable states are exhibited, each containing one of the deterministic local attractors in Figure 1. To this end, define intervals $[a, b]$ and $[c, d]$ such that

$$
0<a<k_{1}^{*}<b<k_{b}<c<k_{2}^{*}<d .
$$

(See Figure 1.) These sets are metastable. Consider first $[a, b]$. By monotonicity of $S, k \leq b$ implies

$$
\mathbf{M}_{\sigma}(k,(b, \infty))=\int_{0}^{\infty} \mathbf{1}_{(b, \infty)}\left[S(k) z^{\sigma}\right] \psi(z) d z \leq \int_{0}^{\infty} \mathbf{1}_{(b, \infty)}\left[S(b) z^{\sigma}\right] \psi(z) d z .
$$

$\overline{7}$ Absorbing sets are also referred to as self-supporting (Futia, 1982, Definition 2.18) or ergodic (Stokey et al., 1989, p. 321). 
Applying a change of variable to the right hand side of this inequality, then,

$$
k \leq b \Longrightarrow \mathbf{M}_{\sigma}(k,(b, \infty)) \leq \int_{p(\sigma)}^{\infty} \psi(z) d z, \quad p(\sigma):=(b / S(b))^{1 / \sigma} .
$$

But $S(b)<b$, as can be seen from Figure 1. Hence $p(\sigma) \rightarrow \infty$ as $\sigma \rightarrow 0$. It can now be deduced from (6) that $\mathbf{M}_{\sigma}(k,(b, \infty))$ converges to zero uniformly for $k \in[a, b]$ as $\sigma \rightarrow 0$. A similar argument shows that, in addition, $\mathbf{M}_{\sigma}(k,(0, a))$ converges to zero uniformly for $k \in[a, b]$ as $\sigma \rightarrow 0$. Now metastability of $[a, b]$ follows from the bound

$$
\begin{aligned}
\sup _{k \in[a, b]} \mathbf{M}_{\sigma}\left(k,[a, b]^{c}\right) & =\sup _{k \in[a, b]}\left[\mathbf{M}_{\sigma}(k,(0, a))+\mathbf{M}_{\sigma}(k,(b, \infty))\right] \\
& \leq \sup _{k \in[a, b]} \mathbf{M}_{\sigma}(k,(0, a))+\sup _{k \in[a, b]} \mathbf{M}_{\sigma}(k,(b, \infty)) .
\end{aligned}
$$

The proof of metastability for $[c, d]$ is similar.

\section{Remaining Proofs}

Proof of Proposition 2 From the standard theory (Futia 1982, Stokey et al. 1989 , Chapter 8$)$, every trajectory $\left(\varphi_{t}\right)$ generated by (3) satisfies the recursion

$$
\varphi_{t+1}(B)=\int \mathbf{M}_{\sigma}(k, B) \varphi_{t}(d k), \quad \forall B \in \mathscr{B}, \quad t=0,1, \ldots
$$

If $t=0, \varphi_{0}$ is interpreted as the degenerate probability concentrated at $k_{0}$.

By way of contradiction, suppose that (3) is globally stable with equilibrium $\varphi^{*}$, and let disjoint nonempty sets $\Lambda_{1}$ and $\Lambda_{2}$ be absorbing. In addition, take $k_{0}^{1} \in \Lambda_{1}$ and $k_{0}^{2} \in \Lambda_{2}$ as two different initial conditions, and let $\left(\varphi_{t}^{1}\right)$ and $\left(\varphi_{t}^{2}\right)$ be their respective trajectories. Using (5) and (7),

$$
\varphi_{1}^{i}\left(\Lambda_{i}\right)=\int \mathbf{M}_{\sigma}\left(k, \Lambda_{i}\right) \varphi_{0}^{i}(d k)=\mathbf{M}_{\sigma}\left(k_{0}^{i}, \Lambda_{i}\right)=1, \quad i=1,2 .
$$

In fact $\varphi_{t}^{i}\left(\Lambda_{i}\right)=1$ is true for any $t$ by induction, which is to say that an economy that starts in $\Lambda_{i}$ remains in $\Lambda_{i}$ for all $t$ with probability one. But $\varphi_{t}^{i} \rightarrow \varphi^{*}$ in total variation norm by hypothesis, and hence setwise (Stokey et al. 1989, Theorem 11.6). Therefore, since $\Lambda_{1}$ and $\Lambda_{2}$ are disjoint,

$$
1 \geq \varphi^{*}\left(\Lambda_{1}\right)+\varphi^{*}\left(\Lambda_{2}\right)=\lim _{t \rightarrow \infty} \varphi_{t}^{1}\left(\Lambda_{1}\right)+\lim _{t \rightarrow \infty} \varphi_{t}^{2}\left(\Lambda_{2}\right)=2
$$

Contradiction. 
Proof of Theorem 1 Stachurski (2002, Theorem 3) shows that any system with multiplicative, lognormal shock such as (3) will be ergodic whenever there exist nonnegative constants $\alpha$ and $C, \alpha<1$, and nonnegative continuous function $V$ on $(0, \infty), \lim _{k \downarrow 0} V(k)=\lim _{k \uparrow \infty} V(k)=\infty$, such that

$$
\mathbb{E} V\left[S(k) \varepsilon^{\sigma}\right] \leq \alpha V(k)+C, \quad \forall k \in(0, \infty)
$$

This bound holds for $V(k):=1 / k+k$. To see this, note that

$$
\mathbb{E} V\left[S(k) \varepsilon^{\sigma}\right]=\frac{1}{S(k)} \mathbb{E}\left(1 / \varepsilon^{\sigma}\right)+S(k) \mathbb{E}\left(\varepsilon^{\sigma}\right)
$$

Consider the first term in the right hand side of (9). By Assumptions 2.2, 2.3 and 2.4, there exists a positive constant $B$ such that

$$
S(k) \geq B f(k) \mathbb{E}\left(1 / \varepsilon^{\sigma}\right) .
$$

Since $B>0, B f$ inherits all of the properties of $f$ in Assumption 2.1. In particular, given any $a_{1} \in(0,1)$, there exists a $\delta>0$ such that

$$
B f(k) \geq k / a_{1}, \quad \forall k \in(0, \delta) .
$$

Also, by monotonicity,

$$
B f(k) \geq B f(\delta), \quad \forall k \in[\delta, \infty)
$$

Combining (10), (11) and (12) gives

$$
\frac{1}{S(k)} \mathbb{E}\left(1 / \varepsilon^{\sigma}\right) \leq a_{1} \frac{1}{k}+C_{1}, \quad \forall k \in(0, \infty)
$$

where $a_{1}<1$ by construction and $C_{1}$ is a finite constant.

Consider now the second term in the right hand side of (9). As savings from wage income is less than total income, and given the restrictions on $f$ and Assumption 2.4, there exist constants $a_{2}$ and $C_{2}$ such that

$$
a_{2}, C_{2} \geq 0, a_{2}<1, \quad \text { and } S(k) \mathbb{E}\left(\varepsilon^{\sigma}\right) \leq a_{2} k+C_{2}, \quad \forall k \in(0, \infty) .
$$

Now let $\alpha:=\max \left(\alpha_{1}, \alpha_{2}\right)$, and $C:=C_{1}+C_{2}$. Using (9), (13) and (14) gives

$$
\mathbb{E} V\left[S(k) \varepsilon^{\sigma}\right] \leq \alpha(1 / k+k)+C=\alpha V(k)+C .
$$

Since $\alpha<1$ and $C$ is finite the condition (8) is satisfied. 


\section{References}

[1] Acemoglu, D. and F. Zilibotti (1997): "Was Prometheus Unbound by Chance? Risk, Diversification and Growth," Journal of Political Economy, 105, 709-751.

[2] Arthur, W. B. (1994): Increasing Returns and Path Dependence in the Economy. Ann Arbor: The University of Michigan Press.

[3] Azariadis, C. and A. Drazen (1990): "Threshold Externalities in Economic Development," Quarterly Journal of Economics, 105, 501-526.

[4] Brock, W. A. and L. Mirman (1972): "Optimal Economic Growth and Uncertainty: The Discounted Case," Journal of Economic Theory, 4, 479-513.

[5] David, P. A. (1997): "Path Dependence and the Quest for Historical Economics: one more Chorus for the Ballad of QWERTY," University of Oxford Discussion Papers in Economic and Social History, 20.

[6] Futia, C. A. (1982): "Invariant Distributions and the Limiting Behavior of Markovian Economic Models," Econometrica, 50, 377-408.

[7] Galor, O. and J. Zeira (1993): "Income Distribution and Macroeconomics," Review of Economic Studies, 60, 35-52.

[8] Huisinga, W. (2001): "Metastability of Markovian Systems: A Transfer Operator Approach in Application to Molecular Dynamics," Ph.D. dissertation, Berlin.

[9] Mackey, M. C., A. Longtin and A. Lasota (1990): "Noise-Induced Global Asymptotic Stability," Journal of Statistical Physics, 60, 735-751.

[10] Quah, D. (1996): "Convergence Empirics Across Economies with (some) Capital Mobility," Journal of Economic Growth, 1, 95-124.

[11] Stachurski, J. (2002): "Economic Dynamical Systems with Multiplicative Noise," Journal of Mathematical Economics, 39 (1-2), 135-152.

[12] Stokey, N. L., R. E. Lucas and E. C. Prescott (1989): Recursive Methods in Economic Dynamics. Massachusetts: Harvard University Press. 